\title{
IoT combined with Block-Chain and 5G infrastructure for its application and development : A Review
}

\author{
Dr. K.Nagarathna rajur
}

Associate Professor

\begin{abstract}
It is a modern type of technology that empowers virtual and physical artefacts to interact and provide digitized services for each other, and includes the Internet of Things (IoT). But it has some benefits, but raises problems of single point of failure, anonymity, accountability, and data integrity due to the new structured design. Challenges like these stands in the path of the introduction of all the most interesting Internet of Things technologies. Bringing the Internet of Things through the public ledger might fix these issues. Decentralized ledger systems are comprised of blockchain as well as distributed ledgers. The introduction of the Internet of Things (IoT) to the blockchain will offer tremendous advantages. Blockchain integration in this paper offers a detailed exploration of how to combine the IoT technology with the IoT scheme. It is followed by reviewing the basic framework and addressing the problems inherent in the system's integration, explaining the advantages of it, and describing ways in which the blockchain can help to overcome such problems. Blockchain as a Service for IoT can illustrate how different protocol concepts can be applied using different service types on blockchain. After this, there would be an important section about the integration of artificial intelligence (AI) into the Internet of Things (IoT) and blockchain. Finally, potential study avenues will focus on ways of applying the Internet of Things (IoT) with blockchain are suggested. The open issues and challenges of 5G-enabled IoT for blockchain-based Industrial automation are also analyzed in the text..
\end{abstract}

Keywords: AI, Block-chain, 5G.

\section{Introduction:-}

Today's Internet of Things is physical and interactive machines communicating with each other as well as individuals that of all manner of objects are able to self-organize and coordinate without human interaction. Most IoT systems solve our day-to-to-day challenges, while the other collects data regarding our physical world and allows for novel services to be created from it. The successful implementation of the Internet of Things is vast, with billions of products deployed in several industries to date [1].

Although the abundance of various advantages that an IoT framework incorporates, including all artefacts connected, handled, and distributed in a single server, there are several difficulties when implementing an IoT architecture in this manner. This technological difficulties standoff could hamper the implementation of potential Internet of Things applications. For starters, a single malfunction, whether the server is down, brings availability and operation to IoT systems to a crashing halt[2]. Additionally, the central server holds all of the IoT device's records, making it a tempting target for attackers. Additionally, protecting data privacy could be questionable when all the more internet of things (IoT) data, which require confidential and personal details, are stored in a single archive, remains under the complete control of a third-party server[3]. The unified design is often suffers from a second dilemma which might not be a feasible answer for the billions of IoT systems which have an annual growth rate of billions[4].

Where more than one error is caused by the use of a centralised IoT architecture, migrating to a decentralised one is more suitable. The blockchain is among the more common and influential distributed ledger technology. A P2P network's ledger records different transactions through several parties in a distributed, shared, and continuously updated database Transactions in a block is organised and allocated to a single ledger entry[3]. The hash function links each block with a timestamp to the preceding one. As such, it is called the blockchain since it establishes chains of links. Only nodes in the consensus agreement can store a transaction in the distributed ledger. The infrastructure behind blockchain encourages knowledge exchange, in which any member of the network has a redundant copy of the gold/genuine ledger that monitors all transactions[5].

IoT integration of blockchain adds several benefits to the table. vulnerability to a single point of failure Additional, as it incorporates state-of-of-the-the-the-the-art authentication, encryption, hash functions, and timestamp, it provides superior protection and privacy. Additionally, the blockchain records static evidence that can't be changed if the rest of the participants 
confirm that [6] For the purpose of this article, we're talking about the industry as a whole, the aim was to address was to have a trustworthy mechanism where the participating IoT devices are the only artefacts to approve or reject a transaction based on their consent [7] The aim of this paper is to introduce the IoT framework, which includes device characteristics and central architecture. In this last section, blockchain is discussed in an informative way that explains the key features and components of the technology. Finally, various use cases of the integration of IoT with blockchain are examined to explain why and see how blockchain solves problems Then, there are a number of aspects of blockchain technologies that are incorporated into blockchain as a business. After this, there would be an important section about the integration of artificial intelligence (AI) into the Internet of Things (IoT) and blockchain. New IoT (intelligent distributed systems) endeavours would be addressed at the conclusion of the study.

This is a good report on combining the latest developments in the Internet of Things (IoT) and blockchain. Both novel and important facets of IoT and IOT are explored in this article, and it is discussed how blockchain may be used for IOT as well as the effects that they have on IOT and IOT. There was no discussion in the survey about the ways that it might be used in the Internet of Things (IoT) sector in the study. They have neglected to talk about incorporating blockchain for the Internet of Things. In addition, the relevant survey neglected to take into consideration the problem of combining AI with IoT, as well as the large amounts of data created by IoT applications, the concerns with scalability for both are much greater.

It may be stated that the authorship of this work is as follows:

- Might help with recent state-of-art analysis and studies on the topic of IoT

- Offering blockchain-as-a-service capabilities into the IoT ecosystem The potential effect of merging AI and IoT on the collaboration between these two industries may be dramatic.

The majority of IoT-based applications are already developed utilising a standardised client-server architecture, cloud storage, a robust database, and the Internet [8,9]. We have identified two significant shortcomings of the IoT unified infrastructure: (i) a single point of failure, which could theoretically bring the whole system down, and (ii) a lack of confidence between the system's institutions [10]. To address the limitations outlined above, decentralised architectures can be used to allow peer-to-peer (P2P) communication between nodes. However, these devices have a number of privacy and security issues, which may enable intruders to initiate a variety of attacks.

There are numerous applications that use IoT devices to improve service quality, including smart homes [11], smart factories [12], smart cities [13], and safe vehicular AdHoc networks [14]. It is the future. However, 5G-based IoT deployments will make use of Blockchain to ensure the protection of IoT applications. There are numerous Blockchain solutions available for protecting IoT networks [15,16], some of which run on fast trustworthy networks and others on insecure networks. As modern blockchain technology runs effectively and exponentially over insecure networks, 5G-enabled IoT devices will use it in real-time. Security will not be the only consideration when using blockchain; it may also be used for data distribution, since it operates in a quicker mode. However, the writers are unaware of an independent statistical proof for the swift solutions. After the statistical evidence is accessible, an ideal world can be created in which quick and trustworthy nodes are linked to the network and can take advantage of $5 \mathrm{G}$ by using specific cloud or fog layers.

\section{IoT}

The Internet of Things (IoT) framework defines a cutting-edge technology that has the potential to transform our lives, businesses, and economies. The Internet of Things generates a plethora of digitised services and applications that provide several advantages over traditional solutions. These applications and services have several features in common, including:

- Sensing capabilities: The wireless sensor network is the primary technology that promotes growth in various IoT domains (WSN). WSNs are usually networks of sensors that detect information about their surroundings and transmit it to a communication medium for processing. Sensors are the IoT building blocks that allow for the collection of both real-time and contextual information about the environment, allowing decision-makers to make accurate and precise decisions in real time.

- Connectivity: one of the main features of the IoT framework that allows billions of devices and items to be accessed remotely. Furthermore, it enables different objects in our environment to be linked and interact with one another through the Internet, allowing the development of new applications and services.

- Large-scale network: as previously reported, the IoT system contains billions of computers, with the number estimated to exceed 75 billion by the end of 2025 [17]. Because of the large number of devices and artefacts, a large-scale network is created that cannot be handled using conventional or traditional methods.

- Dynamic system: The Internet of Things is a dynamic system of nature. It has the ability to attach various objects in various locations. Furthermore, IoT devices can be dynamically adapted to changing circumstances and conditions by using sensors that capture different real-time and contextual information about their surroundings.

- Intelligence capabilities: With advanced hardware, software, and sensing capabilities that allow for the collection of a large amount of contextual data, IoT devices can make wise decisions in a variety of situations and intelligently collaborate with other collaborating objects. 
- Big data: there are billions of IoT computers, resulting in a massive amount of data that cannot be analysed using conventional data mining methods. This is a reference to the phrase "big data." The Internet of Things (IoT) is one of the richest sources of big data, generating a massive amount of data that requires creative analytics approaches to reap the full benefits of IoT data.

- Unique identity: The Internet of Things system allows different objects to communicate over the Internet. The ability to link to the Internet can only be assured if each device has a distinct identity or identifier, such as an IP address. Manufacturers assign a unique identifier to each computer in the IoT scheme, allowing it to upgrade devices to relevant platforms in the event of a security breach. As a result, even though there are billions of IoT products, each one has a distinct identity.

- Autonomous decision: the IoT device contains several sensors that allow for the collection of large amounts of contextual and real-time data about the external environment. These dynamic data allow IoT devices to make context-aware and self-sufficient decisions.

- Heterogeneity: The Internet of Things infrastructure enables various devices and artefacts to be addressed and interact with one another over the Internet. These devices have a variety of features, such as communication protocols, operating systems, platforms, and other software and hardware components. Despite these disparities, the IoT framework enables all of these devices to communicate with one another in an efficient and effective manner.

\section{Blockchain Technology (BC-Technology)}

The word "Blockchain" derives from its technical structure (Chain Blocks), which refers to the connection of each block with the block that comes before it. The blockchain principle interconnects data links or transactions in clusters. The cluster is known as a data structure that contains a large number of financial transactions. Transactions are exchanged between individuals or organisations. These transactions may be financial or non-financial in nature (smart contracts). Any person or organisation that accepts protocol strings and contributes to their development is considered a blockchain participant. The blockchain is not shared by the organisers of these networks or those in charge of software maintenance. The fundamentals of blockchain are covered in this section.

\subsection{Blockchain Fundamentals}

Distributed Ledger Technology created the Blockchain concept (DLT). This technology is being designed to provide Convention validation technology through a network that could span the entire globe in order to promote peer-to-peer transactions as well as all financial transactions. This process marginalises third-party positions in financial transactions such as banks, agents, intermediaries, or any authority that may be needed to ensure and maintain transaction data fulfilment or update. After that, double-check each financial transaction and save it as a new block for an existing transaction. When a transaction is saved inside a string, it cannot be modified, overwritten, or removed, necessitating higher standards of protection and transparency [1]. Figure 1 depicts the primary concept of a blockchain and IoT-based integrated application domain for users.

Figure 1:- Depicts how blockchain technology functions. (3rd)

\subsection{Blockchain's Fundamental Functions}

The Blockchain is an electronic system that uses "Peer to Peer" technology to handle financial transactions. It serves three primary

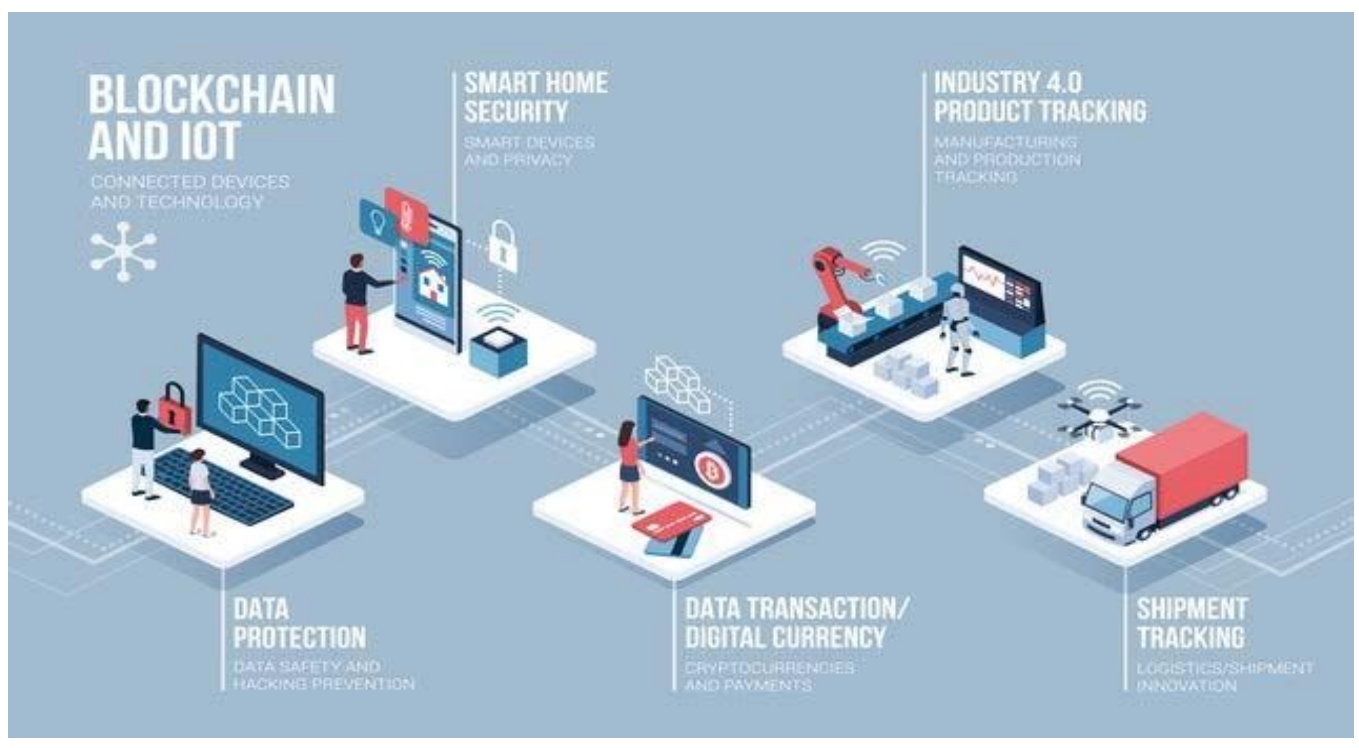

purposes:

1. Allow financial transactions between individuals and institutions all over the world to be highly reliable and secure because they eliminate so-called "double spending." [2] Formalized paraphrase 
2. Allow transaction traceability (the ability to monitor anything on the Internet back to its assets), which means transactions can be clearer, more open, and more secure.

3. Using the same method, protect users from any attacks or abuses by malicious users. Furthermore, there is no need for central authorities to engage in financial markets in order to reduce costs.

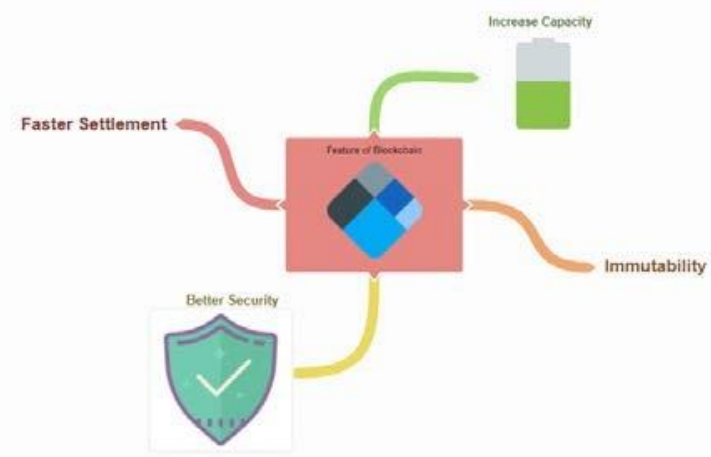

Figure 2: Blockchain Characteristics.

Figure 2 depicts the key characteristics of block chain that make it safer, quicker, and more capable. As shown in Figure 3, a basic blockchain network can be depicted.

Figure 3: Blockchain Network

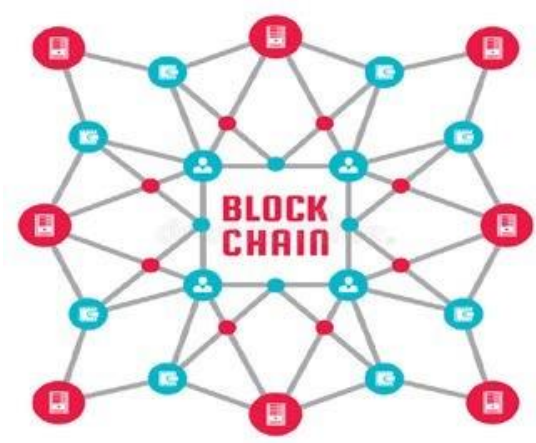

\subsection{Blockchain authorization}

A licenced Blockchain is a network that is used to perform financial transactions by individuals or organisations, such as a group of institutions or banks that monitor financial transactions. This network is limited to delegated to a group of people or organisations that can only access data They can only read and write to the target data. The authorised Blockchain is newer than the permissionless Blockchain. As a result, a licenced Blockchain is a central body, such as a bank, with the power to monitor the rights of individuals and classify them in order for them to engage in the process of reading or writing data. Lesser Permission Blockchain is an open network that anyone or any organisation can access and use. Bitcoin and Ethereum frozen hoops are good examples of unauthorised Blockchain since users can switch to each other without a controller by using bitcoin or ethereum as a payment tool. Since this cluster is open and decentralised, anyone (peer) can access or enter it, and they are free to leave at any time. The counterpart has the right to read or write what has been agreed upon for care. There is no central authority in this network to handle peer interaction, monitor membership, or read or write. The availability of transparency implies that the written material is accessible by all individuals. However, the cryptographic features allow for the development of permission-less Blockchains and the concealment of information pertaining to the privacy of the peers.

\subsection{Block-Chain and IoT}

The Internet of Things (IoT) refers to the interconnection of smart devices for the purpose of gathering data and making decisions. Things can eradicate the lack of protection in Internet objects by integrating Blockchain and Internet objects, where Blockchain includes "security by design." Blockchain features such as incompatibility, openness, readability, data protection, and organisational versatility can be used to address Internet security concerns. The integration of Blockchain and IoT is a promising field of research that has received significant attention but still has many unexplored areas. The comparison between blockchain and IoT is shown in Table 1. 


\begin{tabular}{|l|l|}
\hline Blockchain & IoT \\
\hline Decentralized & Centralized \\
\hline Resource consuming & Resource restricted \\
\hline $\begin{array}{l}\text { Block mining is time- } \\
\text { consuming }\end{array}$ & Demands low latency \\
\hline $\begin{array}{l}\text { Scale poorly with large } \\
\text { network }\end{array}$ & $\begin{array}{l}\text { IoT considered to contains large } \\
\text { number of devices }\end{array}$ \\
\hline $\begin{array}{l}\text { High bandwidth } \\
\text { consumption }\end{array}$ & $\begin{array}{l}\text { IoT devices have limited bandwidth } \\
\text { and resources }\end{array}$ \\
\hline Has better security & $\begin{array}{l}\text { Security is one of the big challenges of } \\
\text { IoT }\end{array}$ \\
\hline
\end{tabular}

Table 1: Comparison Between Blockchain and IoT

\subsection{Blockchain as a Service for IoT:}

After the introduction of Figure 4, we've seen an IoT architecture with a better structure with a service layer consisting of blockchain, which makes it easier to work with Blockchain technology can be used to improve hundreds of industries by reducing the number of middlemen and eliminate unnecessary expenses by using it in a multitude of cases, not only in cryptocurrencies. Since cloud computing may provide various services such as SaaS, PaaS, IaaS, and IaaS, PaaS can be used in IoT applications.

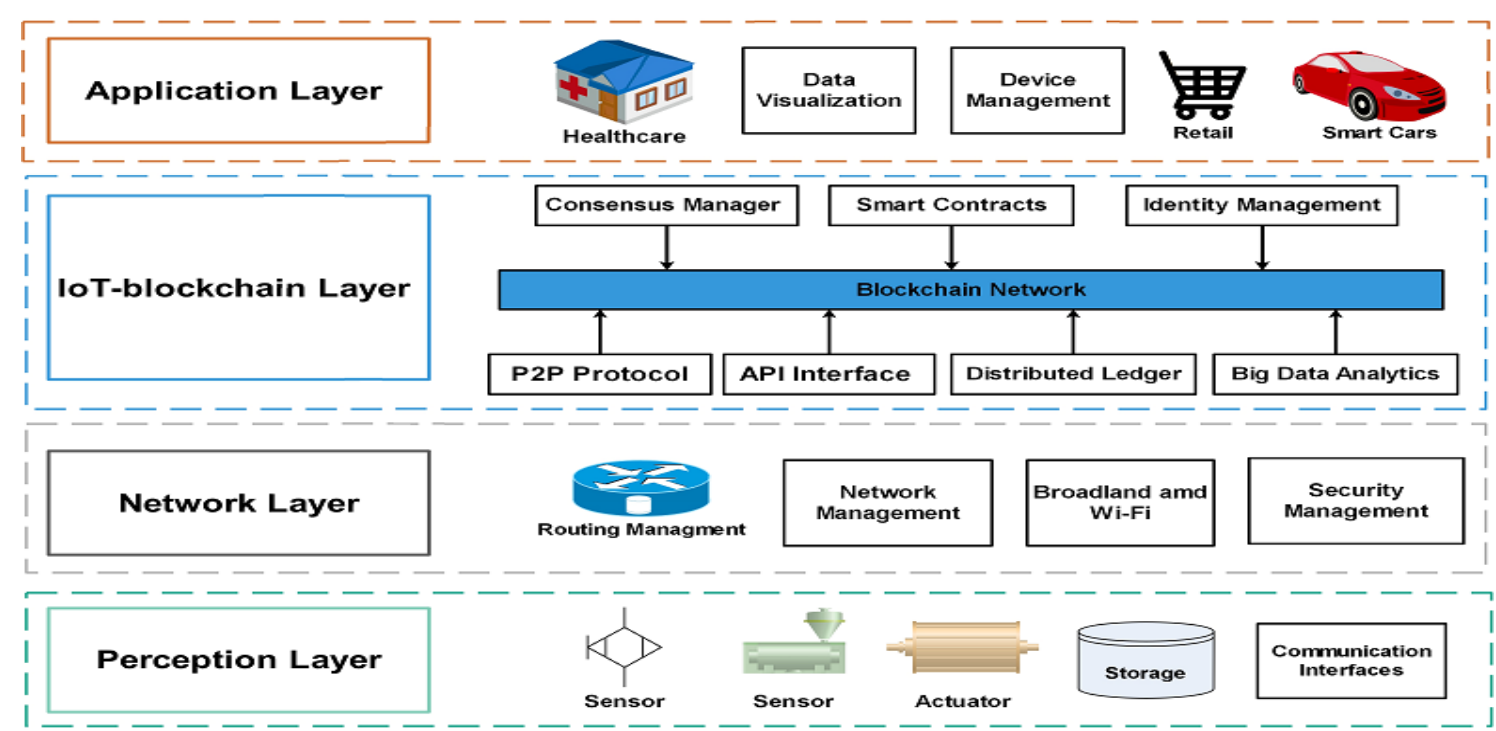

This new initiative furthers the ability to use Blockchain as a Service (BaaS).

Figure 4:- Architecture of IoT with Blockchain

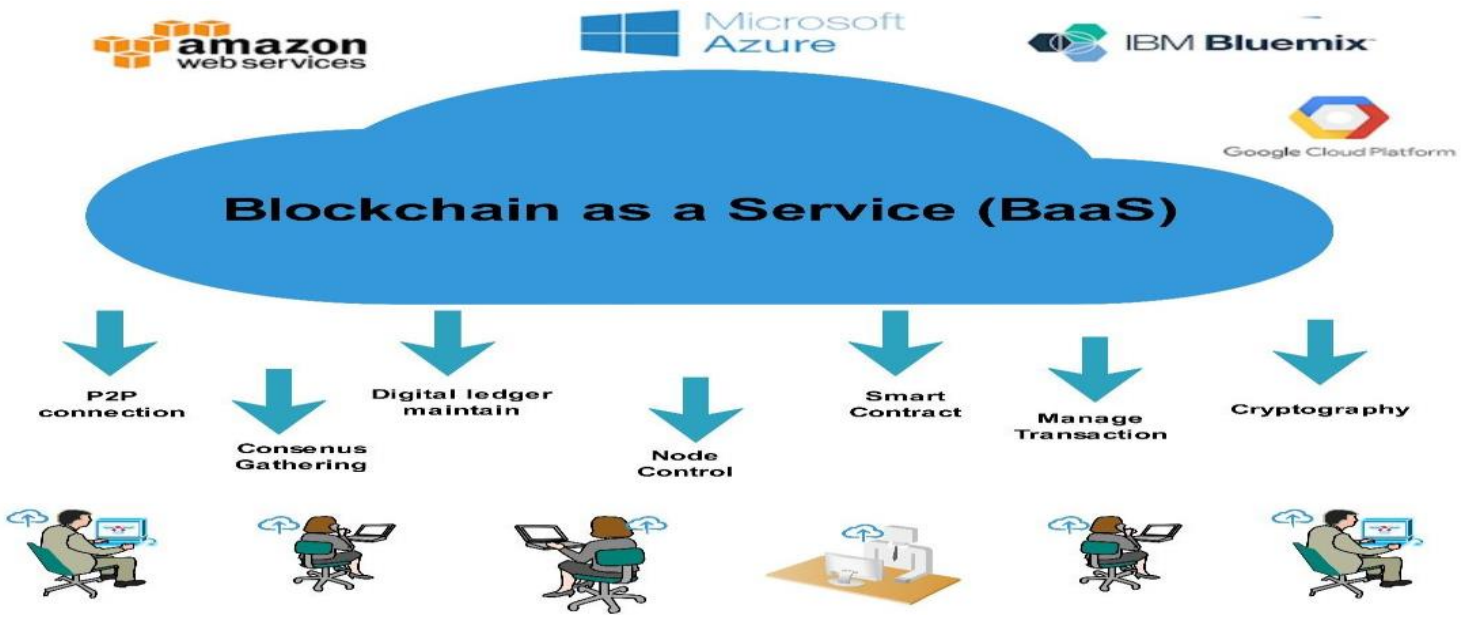

Figure 5:- BaaS as a Blockchain service. 
This is a term used when an organisation aims to extend the use of blockchain to:storing, facilitating, managing, and deploying various features such as smart contracts, decentralisation, incorruptibility, and distributed ledger on cloud computing. As a result, blockchains can support various use cases based on the cloud computing. natural resources These services are needed for fog nodes (IoT devices) to gain the benefits and take advantage of blockchain technology in the IoT setting. PaaS is a service that runs on a cloud. BaaS for IoT devices is known as PaaS, but can also be done indirectly by SaaS. , the initial costs for blockchain implementation are extremely high because it must be created on premise and subsequent costs are greatly diminished because of high performance and infrastructure availability of the distributed ledger Blockchain-as-as-a-a-a-a-service (BaaS) is offered by companies such as Amazon, Microsoft Azure, Oracle, and IBM among others. the graphical design of the six is shown in Figure 5 Although it can be useful for different IoT implementations, BaaS has many benefits, including the ability to expand: On-in our example, we see that platforms that have been designed from scratch can be used to carry out the tasks offered by BaaS more inexpensively and easily. Additionally, BaaS is used alongside other cloud services (SaaSaaS, PaaS, IaaS), which provides an opportunity for blockchain to be more easily integrated with IoT and IIoT applications, and gives rise to an expansion of various combinations of technology. In particular, BaaS delivers additional benefits are greater accessibility and usability. Laying out one of the foundations involves higher ability demands for implementation: In other words, implementation of a blockchain must provide knowledge of cryptographic technology and distributed systems. A BaaS is offered as a one-ready service by the service provider. This allows the customer to put the blockchain in place, deploy, monitor, and run it all without having to know any thing about the technical processes involved.

\section{AI with Blockchain and Internet of Things (IoT)}

It is virtually impossible to dismiss the role that AI has on topics such as capitalism, e-business, healthcare, media, national security, public services, finance, and retailing. as found in the definition of AI in the Merriam-Webster dictionary, AI is referred to as the capacity of a computer to replicate human behaviour There is an urgent need to create new methods and models that use artificial intelligence to implement intelligent activities. AI is most commonly developed and practised by using simulations which use the computer system as a tool for experimentation, or virtual experiments where researchers use the computer system as a testbed the numerous numbers of IoT generate a massive amount of data for AI to contend with a complex technologies like the IoT can provide a sophisticated solution to data integration problems that arise from that many IoT complexity using conventional analytics will not be efficient, so it will be necessary to make use various AI and machine learning methods [18]. With the huge data analysis capabilities like that of AI, various governments and organisations have started to use AI in the quest for IoT data, it has become increasingly common to use it to develop AI methods to gain access to it. Since fog computing has greatly reduced the response time, it will enable real-time based IoT applications to flourish. Thus furthering the integration of AI with IoT may be both an effort to counter external threats and provide an efficient way to foresee those that could be in the system.

On the other hand, combining AI with blockchain helps both technologies, as well as their usages of data across networks. Blockchain can solve many problems for AI, such as removing the ambiguity of AI's ability to be seen. Block-box platforms do not have the potential to have user understanding. With the ledger being distributed to all the network nodes and the process trail of data, data can become more reliable as well-trusted because it is clearly traceable back to the machine[19]. Additionally, blockchain has the potential to enhance data exchange and training which in turn results in better AI models. This can also help lower risk by programming smart contracts to carry out defined actions when the conditions are met [Moreover, it helps to lower risk when programming smart contracts to carry out preprogrammed functions when conditions are met]

Some of the issues that exist in the technology that is being explored by developers of blockchain are able to be addressed by advanced artificial intelligence systems, for instance, consensus mechanisms. Both Proof of Work (PoW) and Proof of Stake (PoS) implementations can enable nodes to do this quickly and reliably. Furthermore, because the mining process uses large amounts of energy, AI techniques that have shown some impact on energy consumption can be successful in blockchain can be incorporated into AI [84] Additionally, federated learning is an example of scalability is not only helps to address issues associated with blockchains, but it also to increase them, as it empowers one to learn simultaneously across the organisation boundaries and locations in real time[20].

Despite the fact that blockchain, artificial intelligence, and IoT are still in the early stages of growth, there are numerous expectations that both for the global economy and our daily lives. The integration of these innovations would offer a new view of our future and a new way of looking at the world. the ecosystem will be augmented by Internet of record IoT will be implemented to handle additional data collection and analysis, which will be updated on our behalf, and its results will be communicated on our behalf and sent to us at regular intervals via smart contracts on the blockchain for time-stamping so we can better track them therefore, so that benefits can be investigated and optimised, further research is needed to investigate and discover the possible and develop the best methods for their use.

The internet infrastructure must be expanded to handle the massive amounts of data processed by large-scale IoT systems. Decentralized or distributed networks with "Peer-to-Peer Networking (PPN), Distributed File Sharing (DFS), and Autonomous Device Coordination (ADC)" capabilities are one of the best ways to solve this. These three functions can be carried out by blockchain, allowing IoT systems to monitor a large number of connected and networked devices. BC enables IoT systems to coordinate the processing of transactions between devices. 


\section{The use of blockchain in $5 \mathrm{G}$-enabled smart industrial automation}

We have discuss about blockchain-based industrial applications in 5G-enabled IoT, which covers existing areas of concern for applications like smart city, smart home, healthcare 4.0, industry 4.0, agriculture, autonomous vehicles, and supply chain management. Blockchain and $5 \mathrm{G}$ are used in both of these applications to enhance encryption, increase capacity, and minimise total operating and capital expenditures, respectively. The subsections that follow provide a more comprehensive overview of these applications.

\subsection{Home automation}

A smart home is a technologically enhanced living environment that seeks to increase the inhabitants' quality of life [21]. It gives the owners protection, ease, and comfort by allowing them to monitor the settings using a smartphone application. Smart home systems use the Internet of Things to communicate with devices and automate those activities based on usage statistics, allowing users to receive real-time, uninterrupted services tailored to their needs. Several research ideas for designing energy-efficient smart homes have been explored in the literature.

\subsection{Intelligent city}

The rising trend of people migrating to cities, combined with the related process of urbanisation, poses a slew of complex challenges for cities' overall infrastructure and their ability to provide residents with basic needs such as water, electricity, transportation, and healthcare. Climate change, population rise, and resource scarcity are all contributing to this exponential urban growth. The concept of a "smart city," which ensures an effective and productive use of available resources by utilising technologies such as IoT and cloud computing, is a constructive answer to these problems. The aim of every smart city is to improve the quality of services provided to residents while lowering the overall operating costs of government [22].

\subsection{Healthcare}

Healthcare is one of the most important aspects of any country's overall growth. It can be seen as a measure of a society's overall well-being. The pressure on modern healthcare systems has increased in recent years as the population and medical conditions have increased. 5G-enabled IoT is being considered as a possible way to relieve the strain on the healthcare system [23]. Remote health monitoring is one of the solutions, which includes using IoT sensor devices to remotely assess and analyse a user's health parameters.

\subsection{The Fourth Industrial Revolution (Industry 4.0)}

The full automation of industrial and business processes has become a reality in the modern age. Massive technological advancements and their application in industry have culminated in the advent of Industry 4.0, a modern approach to manufacturing. It seeks to bring together the strengths of different technical realms, such as the Internet of Things (IoT), Blockchain, and Cyber-Physical Systems (CPS) [24]. IoT is expected to deliver promising transformational solutions to current manufacturing structures as part of Industry 4.0. As a result, it's being hailed as a key enabler for the next wave of advanced industrial automation [25].

\subsection{Logistics and supply chain management}

The supply chain is the web of people, organisations, services, and events that are involved in a product's life cycle. It begins with the production of a product and ends with its sale, including the distribution of raw materials from a supplier to a producer and delivery to the end consumer. A supply chain's typical flow starts with the supplier, then the producer, wholesaler, retailer, and finally the customer. The method for managing resources, documents, and finances as they pass through a supply chain phase is known as supply chain management (SCM) [26]. Given the importance of supply chains, they face a number of problems, including the following [27]:

- $\quad$ Logistic mismanagement

- $\quad$ Lack of visibility and assets

- Improper handling of data

- Inefficient handling of stack

- Ineffective risk management

\subsection{Agriculture.}

Smart agriculture makes use of cutting-edge technology like IoT, GPS, and Big Data to improve the quality and quantity of agricultural products. Temperature, light, soil moisture, and humidity can all be stored in a central control system and analysed using artificial intelligence algorithms [28]. The integration of various technologies in smart agriculture aims to reduce the cost of 
the agricultural supply chain while maintaining product quality. DLTs (Distributed Ledger Technologies) are thought to have the most potential for improving the quality and accountability of these agricultural supply chains [29].

\subsection{Self-driving cars}

The growth of Intelligent Transportation Systems (ITS) has been formidable, thanks to rapid technological advances in sensing, connectivity, analysis, and computation. Smarter, faster, and more accessible transportation facilities and services are now possible thanks to ITS. However, ITS poses a significant security risk because of its propensity toward centralization, which may result in the centralised authorities being temporarily unavailable due to malicious external attacks.

\subsection{Unmanned Aerial Vehicles (UAVs)}

An unmanned aerial vehicle (UAV), also known as a drone, is an airborne device or aircraft that can be controlled remotely by a person or autonomously by an on-board computer [30]. UAV photos can be used in a variety of industries, including urban modelling, surveillance, large-scale mapping, distribution, communications and media, search-and-rescue operations, and agriculture [31]. Several armed forces and civilian organisations use unmanned aerial vehicles (UAVs). To be remotely available, the majority of commercial UAVs depend on Wi-Fi connectivity. Wi-Fi access, on the other hand, is insufficient for contact outside the visual line of sight (LOS). Instead, ubiquitous mobile networks like 5G can be used to operate them outside line-ofsight contact.

\section{Conclusion}

With the centralised IoT architecture posing many challenges, migrating the IoT to one of the distributed ledger technologies could be the best option. The blockchain is one of the most popular forms of distributed ledger technology. It employs a decentralised strategy that improves performance while removing the single point of failure. Furthermore, due to its tamper-proof and immutability functionality, blockchain provides better protection and data integrity. The combination of blockchain and IoT will address problems with the centralised IoT framework while also paving the way for potential innovations. As a result, the aim of this paper was to provide a thorough examination of the IoT system's integration with blockchain technology. The paper provided a detailed discussion of combining IoT with blockchain by highlighting how blockchain addressed IoT problems after outlining the foundations of IoT and blockchain. In addition, recent articles on the integration of IoT and blockchain are discussed. Then, to demonstrate how different features of blockchain technology can be applied as a service for various IoT applications, blockchain as a service for the IoT is addressed. The effect of AI integration on both IoT and blockchain was then discussed. Future research directions in IoT with blockchain were addressed to this end. We provide readers with information about the industrial applications of blockchain in 5G-enabled IoT devices in this article. The discussion is divided into three parts: first, a brief introduction to blockchain, IoT, and $5 \mathrm{G}$, followed by a discussion of relevant industrial applications.

\section{Future Work}

Blockchain further can be used exclusively in the field of encrypted currencies, where it will greatly increase the compatibility of the Internet of things and blockchain. In addition, blockchain can be further explored to integrate with IoT for providing better security and privacy in different smart application domains.

\section{References}

1. Atlam, H.F.; Walters, R.J.; Wills, G.B. Internet of Things: State-of-the-art, Challenges, Applications, and Open Issues. Int. J. Intell. Comput. Res. 2018, 9, 928-938.

2. Atlam, H.F.; Alenezi, A.; Alassafi, M.O.; Alshdadi, A.A.; Wills, G.B. Security, Cybercrime and Digital Forensics for IoT. In Greedoids; Springer Science and Business Media LLC: Berlin, Germany, 2019; Volume 174, pp. $551-577$.

3. Atlam, H.F.; Alenezi, A.; Alassafi, M.O.; Wills, G.B. Blockchain with Internet of Things: Benefits, Challenges, and Future Directions. Int. J. Intell. Syst. Appl. 2018, 10, 40-48.

4. Fernandez-Carames, T.M.; Fraga-Lamas, P. A Review on the Use of Blockchain for the Internet of Things. IEEE Access 2018, 6, 32979-33001.

5. Atlam, H.F.; Wills, G.B. Intersections between IoT and distributed ledger. In Advances in Organometallic Chemistry Volume 60; Elsevier BV: Amsterdam, The Netherlands, 2019; pp. 73-113.

6. Karafiloski, E.; Mishev, A. Blockchain solutions for big data challenges: A literature review. In Proceedings of the IEEE EUROCON 2017-17th International Conference on Smart Technologies, Ohrid, Macedonia, 6-8 July 2017; pp. 763-768.

7. Reyna, A.; Martín, C.; Chen, J.; Soler, E.; Díaz, M. On blockchain and its integration with IoT. Challenges and opportunities. Future Gener. Comput. Syst. 2018, 88, 173-190.

8. Muhammad Habib ur Rehman et al, The role of big data analytics in industrial Internet of Things, Future Gener. Comput. Syst. 99 (2019) 247-259.

9. Hong-Ning Dai et al, Big data analytics for manufacturing internet of things: opportunities, challenges and enabling technologies, Enterprise Inf. Syst. (2019) 1-25. 
10. Rahul Agrawal et al, Continuous security in IoT using blockchain, in: 2018 IEEE International Conference on Acoustics, Speech and Signal Processing (ICASSP), 2018, pp. 6423-6427.

11. S. Tanwar et al, An advanced internet of thing based security alert system for smart home, in: 2017 International Conference on Computer, Information and Telecommunication Systems (CITS), 2017, pp. 25-29.

12. J. Wan et al, A blockchain-based solution for enhancing security and privacy in smart factory, IEEE Trans. Ind. Inf. 15 (6) (June 2019) 3652-3660.

13. Sudeep Tanwar, Sudhanshu Tyagi, Sachin Kumar, The Role of Internet of Things and Smart Grid for the Development of a Smart City, in: Yu-Chen Hu et al. (Eds.), Intelligent Communication and Computational Technologies, Springer Singapore, Singapore, 2018, pp. 23-33.

14. Sudeep Tanwar et al, A systematic review on security issues in vehicular ad hoc network, Secur. Privacy 1 (5) (2018) e39.

15. Sana Moin et al, Securing IoTs in distributed blockchain: analysis, requirements and open issues, Future Gener. Comput. Syst. 100 (2019) 325-343.

16. Hasan Ali Khattak et al, Perception layer security in Internet of Things, Future Gener. Comput. Syst. 100 (2019) 144164.

17. Statista. Internet of Things (IoT) Connected Devices Installed Base Worldwide from 2015 to 2025 (in Billions). 2018. Available online:https: //www.statista.com/statistics/471264/iot-number-of-connected- devices-worldwide/ (accessed on 8 October 2020).

18. Alzahrani, A.G.; Alenezi, A.; Atlam, H.; Wills, G.B. A Framework for Data Sharing between Healthcare Providers using Blockchain. In Proceedings of the 5th International Conference on Internet of Things, Big Data and Security (IoTBDS 2020), Prague, Czech Republic, 7-9 May 2020; pp. 349-358.

19.Baldominos, A.; Saez, Y. Coin.AI: A Proof-of-Useful-Work Scheme for Blockchain-Based Distributed Deep Learning. Entropy 2019, 21, 723.

20.Bilodeau, S. Massive Computing for Bitcoin Mining and AI. Available online:https: //towardsdatascience.com/energy-smartbitcoin-mining-dd7bd2d2a3fa(accessed on 8 October 2020).

21. Knud Erik Skouby, Per Lynggaard, Smart home and smart city solutions enabled by 5G, IoT, AAI and CoT services, in: Contemporary Computing and Informatics (IC3I), 2014 International Conference on, pp. 874-878.

22. Andrea Zanella et al, Internet of things for smart cities, IEEE Internet Things J. 1 (1) (2014) 22-32.

23. Aparna Kumari et al, Fog computing for Healthcare 4.0 environment: opportunities and challenges, Comput. Electr. Eng. 72 (2018) 1-13.

24. Wattana Viryasitavat et al, Blockchain-based business process management (BPM) framework for service composition in industry 4.0, J. Intell. Manuf. (2018) 1-12.

25. Li Da Xu, Eric L. Xu, Ling Li, Industry 4.0: state of the art and future trends, Int. J. Prod. Res. 56 (8) (2018) $2941-2962$.

26. What is supply chain (SC)? URL:https://whatis.techtarget.com/definition/supply-chain (visited on 02/17/2019).

27. Sneha S. Kothari, Simran V. Jain, Abhishek Venkteshwar, The Impact of IOT in Supply Chain Management, 2018.

28. Jun Lin, et al., Blockchain and IoT based food traceability for smart agriculture, in: Proceedings of the 3rd International Conference on Crowd Science and Engineering, ACM, 2018, p. 3.

29. M. Tripoli, J. Schmidhuber, Emerging opportunities for the application of blockchain in the agri-food industry, in: FAO and ICTSD: Rome and Geneva. Licence: CC BY-NC-SA 3, 2018.

30 Applications of Unmanned Aerial Vehicle (UAV) based Remote Sensing in NE Region - ISRO. URL:https://www.isro.gov.in/applications-of-unmanned- aerial-vehicle-uav-based-remote-sensing-ne-region.

31. Sathyanarayanan Chandrasekharan et al, Designing and implementing future aerial communication networks, IEEE Commun. Mag. 54 (5) (2016) 26- 34. 